\title{
Comparison of different chemoradiotherapy regimens for the preoperative treatment in patients with locally advanced rectal cancer: a network meta-analysis
}

\author{
Zhengyi Yu ${ }^{1 \#}$, Jiawei Wang ${ }^{1 \#}$, Lingyan $\mathrm{Xu}^{1 "}$, Jin Liu ${ }^{2}$, Xiaofeng Chen ${ }^{1}$, Yanhong Gu ${ }^{1}$ \\ ${ }^{1}$ Department of Oncology, ${ }^{2}$ Clinical Medicine Research Institution, The First Affiliated Hospital of Nanjing Medical University, Nanjing, China \\ Contributions: (I) Conception and design: Z Yu; (II) Administrative support: Y Gu, X Chen; (III) Provision of study materials or patients: Z Yu; (IV) \\ Collection and assembly of data: Z Yu, J Wang, L Xu; (V) Data analysis and interpretation: Z Yu, J Liu; (VI) Manuscript writing: All authors; (VII) \\ Final approval of manuscript: All authors. \\ "These authors contributed equally to this work. \\ Correspondence to: Yanhong Gu, MD, PhD. Department of Oncology, The First Affiliated Hospital of Nanjing Medical University, 300 Guangzhou \\ Rd, Nanjing 210029, China. Email: guyhphd@163.com; Xiaofeng Chen, MD. Department of Oncology, The First Affiliated Hospital of Nanjing \\ Medical University, 300 Guangzhou Rd, Nanjing 210029, China. Email: chenxiaofengnjmu@163.com.
}

Background: The efficacy of different neoadjuvant chemoradiotherapy regimens on locally advanced rectal cancer (LARC) remains confusing. We evaluated them together via a network meta-analysis in terms of survival benefits to find the optimal treatment.

Methods: We searched, EMBASE, Cochrane Central Register of Controlled Trials and the ClinicalTrials website according to the selection criteria for eligible publications before Oct 25, 2019. Pathological complete response rate (pCR), disease-free survival (DFS), and overall survival (OS) were analyzed based on Bayesian methods in the meta-analysis.

Results: Twenty-five articles containing 7,142 participants and 12 preoperative regimens were analyzed. In terms of pCR, radiation therapy plus 5-fluorouracil $(\mathrm{RT}+5-\mathrm{Fu})$, RT plus capecitabine (RT+CAPE), RT plus 5-fluorouracil and oxaliplatin (RT+FOLFOX), RT plus capecitabine and oxaliplatin (RT+XELOX), and RT plus S-1 and irinotecan (RT+IS) were better than RT alone [odds ratio (OR) $=2.66,95 \%$ credible interval [CrI], 1.38-5.01; OR =3.11, 95\% CrI: 1.33-6.98; OR =4.03, 95\% CrI: 1.77-9.47; OR =4.22, 95\% CrI: $1.60-$ 10.87; OR =4.55, 95\% CrI: 1.11-18.88, respectively] and RT+FOLFOX and RT+XELOX were superior to FOLFOX (OR =4.58, 95\% CrI: 1.57-14.19; OR =4.81, 95\% CrI: 1.20-18.73), too. Benefits could be seen on comparing RT+CAPE, RT+FOLFOX, and RT+XELOX with RT (OR =0.84, 95\% CrI: 0.73-0.97; OR $=0.88,95 \%$ CrI: $0.80-0.97$; OR $=0.79,95 \%$ CrI: 0.66-0.95, respectively) in DFS. RT+XELOX seemed to have better effects on OS compared than RT+5-Fu and RT+CAPE (OR =0.78, 95\% CrI: 0.61-1.00; OR $=0.86$, 95\% CrI: $0.74-1.00$, respectively). Moreover, according to surface under the cumulative ranking curve analysis, RT+XELOX had the best outcomes in terms of pCR (79.18\%) and OS (83.49\%) and RT plus capecitabine, irinotecan, and cetuximab (RT+XELIRI+CET) ranked first with respect to DFS (87.86\%).

Conclusions: RT+XELOX is likely to be the best treatment with a comprehensive curative effect and the standard treatment of 5-fluorouracil-based chemoradiotherapy has some advantages, as well. More relevant evidence is needed for clinicians' guidance.

Keywords: Chemoradiotherapy; locally advanced rectal cancer (LARC); neoadjuvant treatment; network metaanalysis; preoperative treatment

Submitted Jan 19, 2020. Accepted for publication Jun 23, 2020.

doi: $10.21037 /$ tcr-20-683

View this article at: http://dx.doi.org/10.21037/tcr-20-683 


\section{Introduction}

Locally advanced rectal cancer (LARC) is a specific rectal cancer with T3-4, N0 or T any, N1-2 stage estimated by magnetic resonance imaging (MRI). Fluorouracil-based chemotherapy and radiotherapy are currently recommended as the standard preoperative neoadjuvant therapies for LARC (1). To achieve maximum clinical benefits, clinicians have made several attempts to combine different chemotherapy drugs. However, no conclusion has been reached to date. Due to the lack of sufficient information to comprehensively and directly compare different schemes, we performed a Bayesian network meta-analysis to directly and indirectly compare different interventions in LARC by determining the pathological complete response rate (pCR), disease-free survival (DFS), and overall survival (OS). Moreover, we revealed the results of ranking different regimens for LARC to guide clinical practice. We present the following article in accordance with the PRISMA guideline checklist (available at http://dx.doi.org/10.21037/ tcr-20-683).

\section{Methods}

This meta-analysis adheres to the requirements of the Preferred Reporting Items for Systematic Reviews and Meta-Analyses (PRISMA) statement. An IRB approval and informed consent of the patients were not required for this network meta-analysis.

\section{Search strategy}

Databases including, EMBASE, Cochrane Central Register of Controlled Trials (CENTRAL2019), and the ClinicalTrials website (https://clinicaltrials.gov) were explored to identify eligible articles before Oct 25, 2019 with no language restrictions. The keywords included "(colorectal or rectum or rectal) and (cancer or carcinoma or tumor) and (neoadjuvant or preoperative) treatment", "clinical trials" and the list of the relevant therapeutic drugs and measures. Two authors (ZYY and LYX) independently picked out relevant articles by screening the titles and abstracts according to the selection criteria. Only trials with full-text articles were enrolled. Different articles belonging to the same trials were reserved and the newest outcomes were extracted. Discrepancies were resolved by discussion.

\section{Selection criteria}

We analyzed the articles meeting the following inclusion criteria:

(I) Randomized controlled trials (RCTs) or qualified cohort studies;

(II) Comparison of different preoperative treatments for LARC;

(III) Twelve preoperative regimens were enrolled, namely, radiation therapy plus 5 -fluorouracil (RT+5-Fu); RT plus capecitabine (RT+CAPE); RT plus 5 -fluorouracil and oxaliplatin (RT+FOLFOX); RT plus capecitabine and oxaliplatin (RT+XELOX); RT plus capecitabine and irinotecan (RT+XELIRI); RT plus S-1 and irinotecan (RT+IS); RT plus oral tegafur (RT+UFT); RT plus capecitabine, irinotecan, and cetuximab (RT+XELIRI+CET); 5 -fluorouracil and oxaliplatin (FOLFOX); RT plus capecitabine and bevacizumab (RT+CAPE+BEV); RT; RT plus 5 -fluorouracil and irinotecan (RT+FOLFIRI). At least two different regimens were compared and at least one agent should be chemoradiotherapy;

(IV) Each arm of different trials must apply only one kind of treatment;

(V) Clinical outcomes included pCR and hazard ratio (HR) with $95 \%$ confidence interval (CI) of DFS and OS.

Studies would be excluded if:

(I) The trial only had one treatment regimen or a single arm received more than one kind of regimen;

(II) The publication type was a case-control study or matching research;

(III) No sufficient data.

\section{Data extraction and quality assessment}

The following contents were extracted: first author's name, publication year, treatment regimens, events and sample size of pCR, HR with $95 \%$ CI of DFS and OS, and median follow-up time if provided.

The quality of the eligible articles was assessed separately based on the different types of trials. The RCTs bias was evaluated according to the Cochrane Handbook for Systematic Reviews of Interventions (2). Only one cohort study was assessed using the Newcastle-Ottawa Scale 
(NOS) (3).

\section{Statistical analysis}

We performed a network meta-analysis based on Bayesian methods $(4,5)$. Odds ratio (OR) and $95 \%$ credible interval (95\% CrI) were calculated for pCR using GeMTC version-0.14.3. The consistency model was firstly chosen for analysis. There were totally 300,000 times simulated interactions (75,000 per chain). Annealing value was set at 100,000 . The consistency was assessed by the node-splitting method. The method separated the evidence concerning certain comparison into direct and indirect evidence, and the inconsistency was reported as the Bayesian P value (6). If any evidence of inconsistency was observed, we would turn to use the inconsistency model.

HR and $95 \%$ CrI were calculated for DFS and OS using OpenBUGS321. The fixed-effects model was used for analysis. A total of 300,000 simulated iterations were updated (100,000 per chain). Annealing value was set at 100,000 . The convergence of iterative simulations was estimated by Gelman-Rubin-Brook diagrams $(7,8)$. If the convergence was not satisfied, we would increase the operation time. If problems remained, we would reappraise the included data or switch to add the random-effects model.

The rank probabilities would be sorted in plots. And the global effectiveness of each treatment would be ranked by the surface under the cumulative ranking curve analysis (SUCRA) (9).

The network structure diagrams, funnel plots and Begg's test were all completed with STATA14. If the publication bias existed, we would check the outcomes of all publications and find the source of bias. Trim and fill methods might be applied to solve the bias (10).

\section{Results}

\section{Search results and study characteristics}

By reviewing the titles and abstracts and assessing full articles based on the selective criteria, 25 articles containing a total of 20 trials and a total of 7,142 participants were included in the network meta-analysis. The characteristics of the 20 studies included are listed in Table 1 (11-35). Among them, 19 studies were RCT and one was a cohort study. The main reasons for including only one cohort study were multiple-arm study and strong relevance to this topic. There were no significant risks of bias found in the articles included. The network structure diagrams are detailed in Figure 1, which is the reflection of the relationships of different preoperative treatment regimens. Meanwhile, the number of comparisons was expressed proportionally by the thicknesses of the lines, and the number of treatments was reflected proportionally in the diameters of the circles. Figure 2 provides the ORs and the corresponding 95\% CrIs of pCR as well as the HRs and the corresponding 95\% CrIs of DFS and OS. The rank probability of each regimen is displayed in Figure 3. Value and plots of SUCRA are summarized in Figure 4. We would introduce these results from three aspects: pCR, DFS and OS, respectively.

\section{$p C R$}

All studies except the NCT0002523 trial conducted by Bosset et al. (26) reported pCR of all 12 different treatment regimens and a total of 6,000 participants were enrolled in the analysis of pCR. The pCR ranged from $2.5 \%$ to $27.5 \%$. The details of the comparison between all 12 treatments are displayed in Figure 1A. The ORs and the corresponding 95\% CrIs of pCR are shown in Figure 2A. The patients receiving $\mathrm{RT}+5-\mathrm{FU}, \mathrm{RT}+\mathrm{CAPE}, \mathrm{RT}+\mathrm{FOLFOX}$, $\mathrm{RT}+\mathrm{XELOX}$, and RT+IS were significantly superior than $\mathrm{RT}$ in $\mathrm{pCR}(\mathrm{OR}=2.66,95 \% \mathrm{CrI}: 1.38-5.01$; $\mathrm{OR}=3.11$, 95\% CrI: $1.33-6.98$; OR $=4.03$, 95\% CrI: $1.77-9.47$; OR $=4.22,95 \%$ CrI: $1.60-10.87$; OR $=4.55,95 \%$ CrI: $1.11-$ 18.88; respectively). And the pCR of RT+FOLFOX and RT+XELOX statistically preceded FOLFOX $(\mathrm{OR}=4.58$, 95\% CrI: 1.57-14.19; OR =4.81, 95\% CrI: $1.20-18.73$ ). The summarized possibility value of the rankings for these treatments in Figure $3 A$ is a direct plot of rank probability. Figure $4 A$ is the plot of SUCRA for each intervention and its detailed values. According to the results above, the regimens from best to worst were RT+XELOX, RT+IS, RT+FOLFOX, RT+CAPE, RT+FOLFIRI, $\mathrm{RT}+\mathrm{UFT}, \mathrm{RT}+5-\mathrm{FU}, \mathrm{RT}+\mathrm{CAPE}+\mathrm{BEV}, \mathrm{RT}+\mathrm{XELIRI}$, $\mathrm{RT}+\mathrm{XELIRI}+\mathrm{CET}$, RT, and FOLFOX.

\section{DFS}

Twelve studies including nine preoperative treatment regimens $(\mathrm{RT}+5-\mathrm{FU}, \mathrm{RT}+\mathrm{CAPE}, \mathrm{RT}+\mathrm{FOLFOX}$, $\mathrm{RT}+\mathrm{XELOX}, \mathrm{RT}+\mathrm{XELIRI}, \mathrm{RT}+\mathrm{IS}, \mathrm{RT}+\mathrm{UFT}$, $\mathrm{RT}+\mathrm{XELIRI}+\mathrm{CET}, \mathrm{RT})$ were allocated for the analysis of DFS (5,052 participants). Their relationship to different treatments is detailed in Figure $1 B$ and the pooled HRs and 
Table 1 Characteristics of the studies included

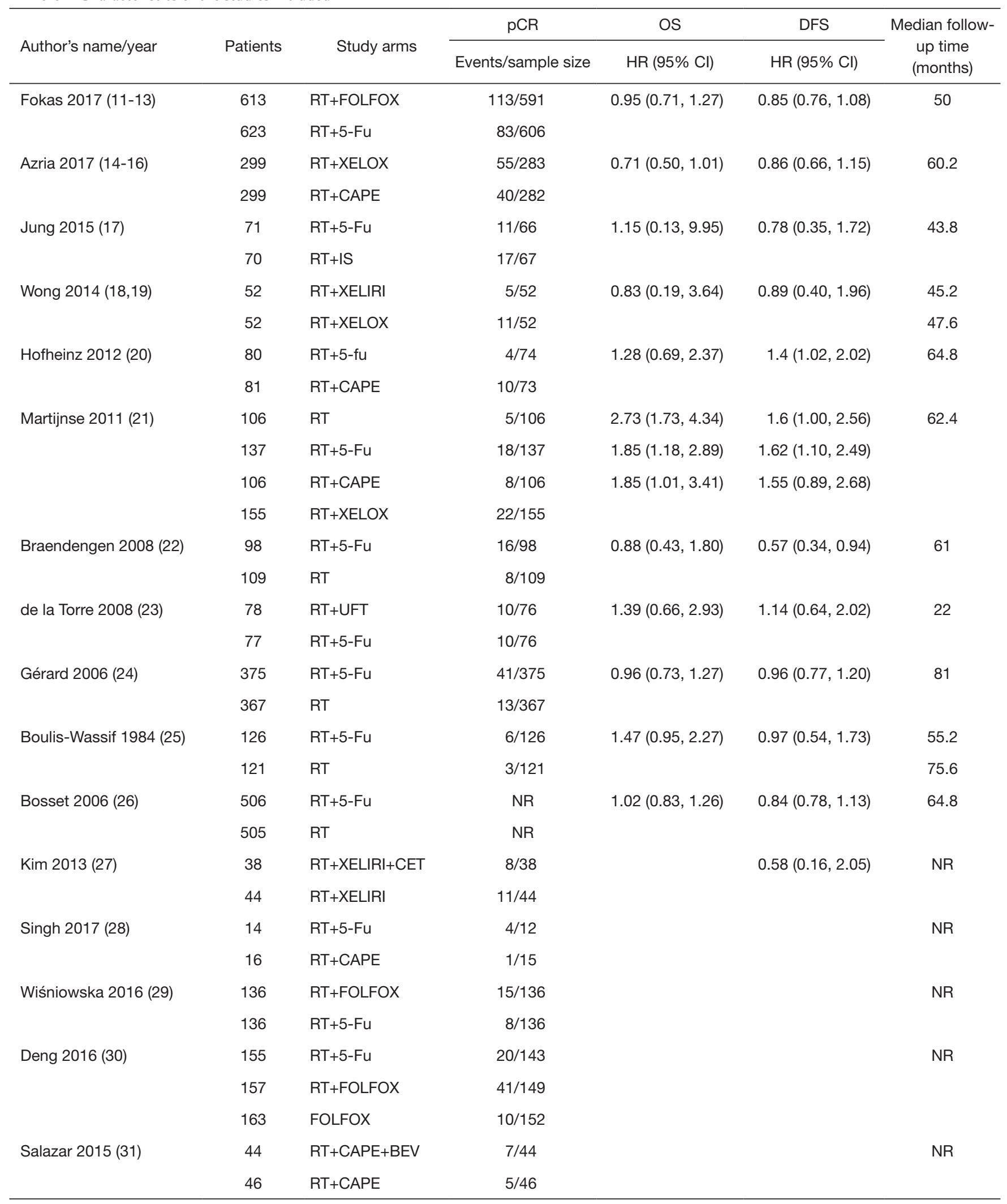

Table 1 (continued) 
Table 1 (continued)

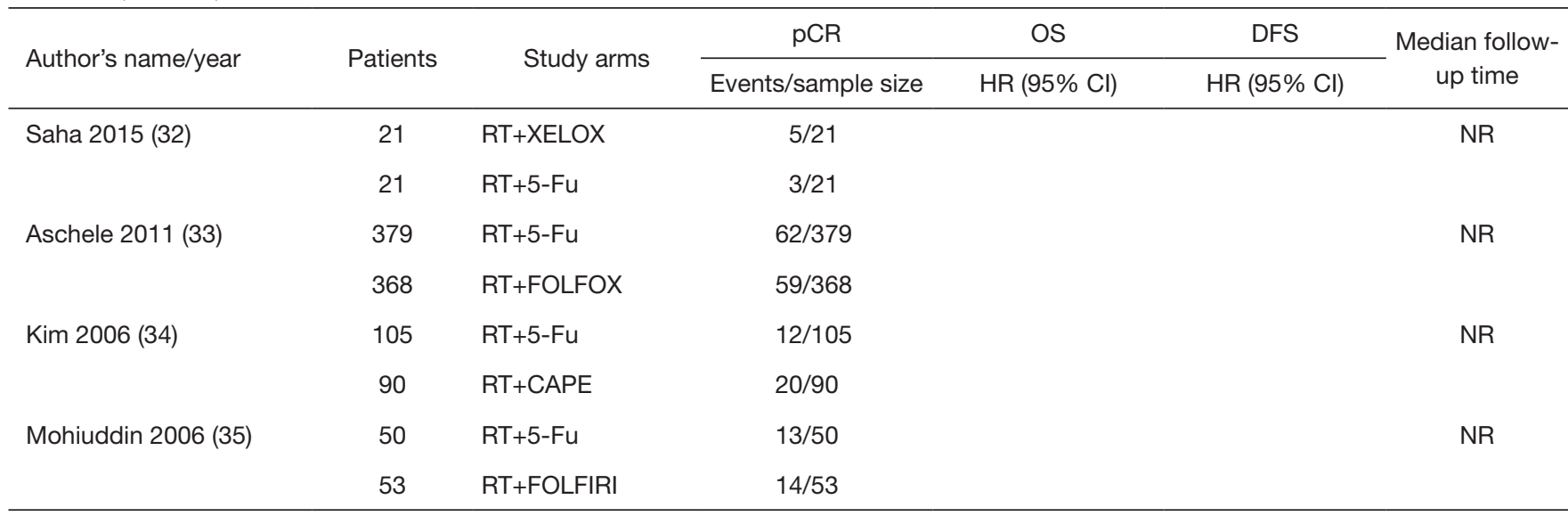

$\mathrm{RT}+5-\mathrm{Fu}$, radiation therapy plus 5-fluorouracil; $\mathrm{RT}+\mathrm{CAPE}$, radiation therapy plus capecitabine; $\mathrm{RT}+\mathrm{FOLFOX}$, radiation therapy plus 5-fluorouracil and oxaliplatin; RT+XELOX, radiation therapy plus capecitabine and oxaliplatin; RT+XELIRI, radiation therapy plus capecitabine and irinotecan; RT+IS, radiation therapy plus $\mathrm{S}-1$ and irinotecan; RT+UFT, radiation therapy plus oral tegafur; $\mathrm{RT}+\mathrm{XELIRI+CET}$, radiation therapy plus capecitabine, irinotecan and cetuximab; FOLFOX, 5-fluorouracil and oxaliplatin; RT+CAPE+BEV, radiation therapy plus capecitabine and bevacizumab; RT, radiation therapy; RT+FOLFIRI, radiation therapy plus 5-fluorouracil and irinotecan; NR, not reported; HR, hazard ratio; $\mathrm{Cl}$, confidence interval.

corresponding $95 \%$ CrIs of DFS is shown in Figure $2 B$. $\mathrm{RT}+\mathrm{CAPE}, \mathrm{RT}+\mathrm{FOLFOX}$, and RT+XELOX had a better DFS compared with RT (HR $=0.84,95 \%$ CrI: 0.73-0.97; HR $=0.88$, 95\% CrI: $0.80-0.97$; HR $=0.79,95 \%$ CrI: $0.66-$ 0.95 , respectively). According to the rank probabilities and SUCRA, RT+XELIRI+CET, RT+XELOX, RT+XELIRI, $\mathrm{RT}+\mathrm{CAPE}, \mathrm{RT}+\mathrm{FOLFOX}, \mathrm{RT}+5-\mathrm{FU}, \mathrm{RT}+\mathrm{UFT}$, $\mathrm{RT}+\mathrm{IS}$, and RT indicated a decreasing tendency of DFS (Figures 3B,4B).

\section{OS}

The results of OS were calculated from 11 studies with a total of 4,970 participants including eight kinds of preoperative treatment regimens $(\mathrm{RT}+5-\mathrm{FU}, \mathrm{RT}+\mathrm{CAPE}$, $\mathrm{RT}+\mathrm{FOLFOX}, \mathrm{RT}+\mathrm{XELOX}, \mathrm{RT}+\mathrm{XELIRI}, \mathrm{RT}+\mathrm{IS}$, $\mathrm{RT}+\mathrm{UFT}, \mathrm{RT})$. The relationship of different regimens is detailed in Figure $1 C$ and the efficacy of different therapeutic paradigms for HRs and corresponding $95 \%$ CrIs is detailed in Figure $2 C$. As indicated in the results, no strong survival benefit could be seen in the direct comparisons. The RT+XELOX seemed to have better effects in OS compared with $\mathrm{RT}+5-\mathrm{Fu}$ and $\mathrm{RT}+\mathrm{CAPE}(\mathrm{OR}=0.78,95 \% \mathrm{CrI}$ : 0.61-1.00; OR $=0.86,95 \%$ CrI: 0.74-1.00, respectively). Based on the results of rank probabilities and SUCRA, the treatment regimens from best to worst were RT+XELOX, $\mathrm{RT}+\mathrm{XELIRI}, \mathrm{RT}+\mathrm{CAPE}, \mathrm{RT}+\mathrm{IS}, \mathrm{RT}+\mathrm{FOLFOX}, \mathrm{RT}$,
$\mathrm{RT}+5-\mathrm{FU}$, and RT+UFT (Figures 3C,4C).

\section{Publication bias}

The funnel plots are displayed in Figure 5. The funnel plots were symmetric. The publication bias was not statistically significant as evaluated using Begg's test ( $\mathrm{pCR}, \mathrm{z}=0.71$, $\mathrm{P}=0.481 ; \mathrm{DFS}, \mathrm{z}=0.33, \mathrm{P}=0.743 ;$ OS, $\mathrm{z}=0.98, \mathrm{P}=0.360$ ).

\section{Discussion}

This study is a network meta-analysis that compared the efficacy of single preoperative treatments for LARC. Twenty studies with a total of 7,142 participants were included in the final analysis and 12 regimens were involved in the comparison as described previously. We compared the efficacy of different regimens from three aspects (pCR, DFS, OS). Based on the rank probabilities and SUCRA, RT + XELOX had superiority on pCR and OS. This treatment regimen captured preponderance in DFS with a second place likewise.

At present, radiotherapy combined with chemotherapy is a consensus on the preoperative treatment of LARC. A Cochrane review including 6 RCTs found that chemotherapy added to preoperative radiation in patients with LARC reduced the risk of local recurrence, but had no effect on OS, 30-day mortality, sphincter preservation, and late toxicity (36). Our results revealed that the benefit of 

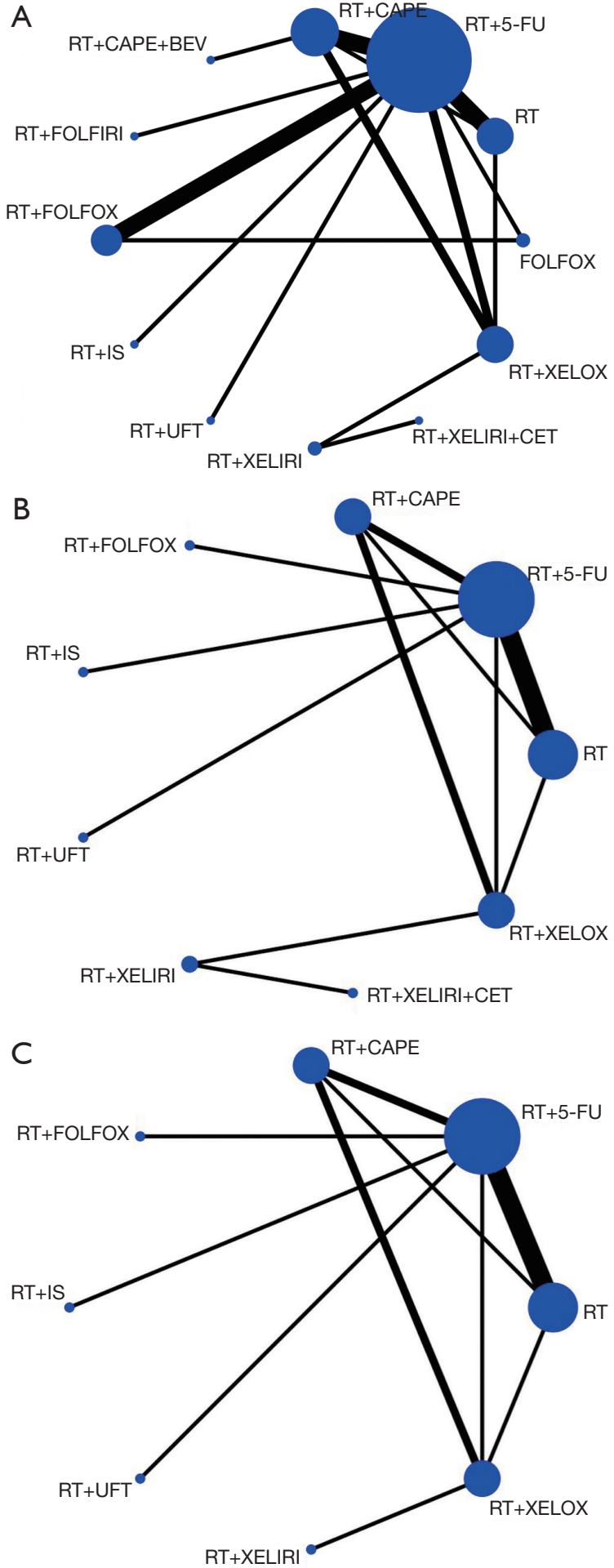

Figure 1 Network of comparisons of pCR, (A), DFS (B) and OS (C) for meta-analysis. The size of the circle indicates the number of participants in each treatment. The width of lines indicates the number of direct comparisons between two treatments. pCR, pathological complete response rate; DFS, disease-free survival; OS, overall survival. radiotherapy alone in terms of pCR and PFS was obviously inferior to preoperative concurrent chemoradiotherapy and provided an evidence to support standard treatment.

In the development of clinical practice, clinicians found that long-course and short-course radiation in neoadjuvant treatment had different advantages. Long-course radiation mainly had a higher pCR than short-course radiation. Further, they suspected that the reason for this was the duration between surgical interval and neoadjuvant therapy. Scholars also found that a surgical interval longer than 6 to 8 weeks from the end of neoadjuvant CRT and surgery significantly improved the pCR from $13.7 \%$ to $19.5 \%$ in a meta-analysis $(37,38)$. In the researches included in our analysis, only few patients in Wiśniowska's study underwent short-course radiation (29). Long-course radiation was used in most studies but the dose was a little lower in an individual early study (34.5 Gy) (25). In the aspect of surgical intervention, some articles did not mention it (11-16,20,21, $27,29,30,32)$. Moreover, the rest of the studies reported the interval to be 3-10 weeks. These data were not specific in the subgroup analysis and the influence on the outcomes was assessed as unknown. With regard to preoperative chemotherapy regimens, the standard treatment is based on fluorouracil. 5-FU, capecitabine, UFT, and S-1 all belong to fluorouracil drugs. According to the results of our network analysis, $\mathrm{RT}+\mathrm{CAPE}$ is superior to $\mathrm{RT}+5-\mathrm{FU}$ and RT+UFT in terms of all three aspects. However, NSABP R-04 has shown that capecitabine was equivalent to 5 -FU in perioperative chemoradiotherapy. There were no differences in locoregional events, DFS, OS, pCR, and surgical downstaging $(39,40)$. Differently, in Hofheinz's study, capecitabine had better results than 5-FU. However, the original non-inferiority design made it unable for capecitabine to replace 5-FU totally (20). Further superiority trials would not be carried out. These facts could also explain the reasons for RT+XELOX possibly being the best regimen. Commonly, RT+XELOX was slightly better than RT+CAPE, but there was no obvious statistical difference each time. After being processed by the passalong effect of the chains in meta-analysis, the advantages could finally become more obvious.

Fluorouracil-based chemotherapy and radiotherapy could decrease the local recurrence rate, but it had no benefit to DFS and OS. Hence, the mixture of chemotherapy drugs is likely to have the potential to control the distant metastasis for enhanced survival. In our meta-analysis, RT+XELOX regimen had striking rankings in $\mathrm{pCR}$ and $\mathrm{OS}$ compared with RT+CAPE and RT+5-Fu. However, based on the 
A

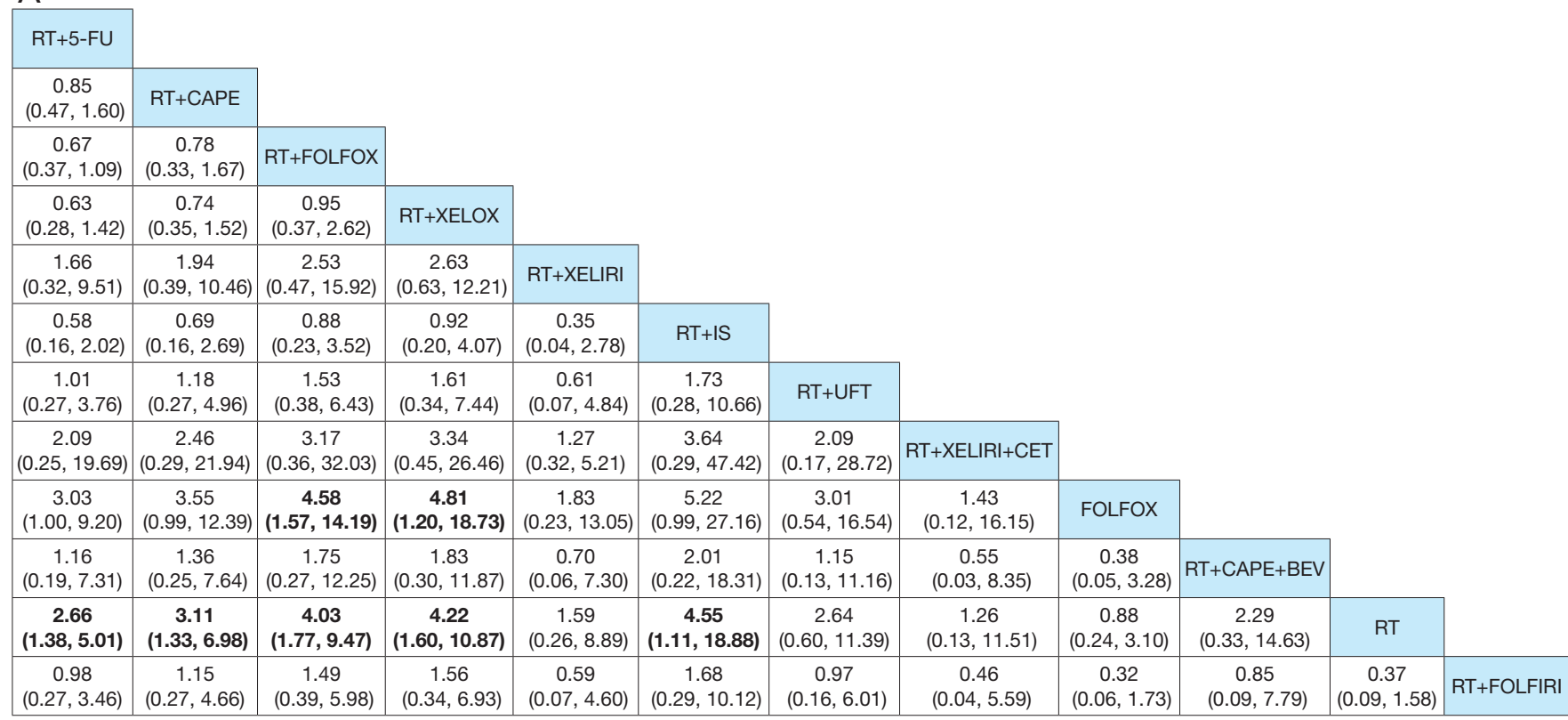

B

\begin{tabular}{|c|c|c|c|c|c|c|c|c|}
\hline $\mathrm{RT}+5-\mathrm{FU}$ & & & & & & & & \\
\hline $\begin{array}{c}0.90 \\
(0.79,1.02)\end{array}$ & $\mathrm{RT}+\mathrm{CAPE}$ & & & & & & & \\
\hline $\begin{array}{c}0.93 \\
(0.86,1.01)\end{array}$ & $\begin{array}{c}1.04 \\
(0.90,1.21)\end{array}$ & RT+FOLFOX & & & & & & \\
\hline $\begin{array}{c}0.84 \\
(0.70,1.00)\end{array}$ & $\begin{array}{c}0.94 \\
(0.83,1.06)\end{array}$ & $\begin{array}{c}0.90 \\
0.74,1.09)\end{array}$ & RT+XELOX & & & & & \\
\hline $\begin{array}{c}0.80 \\
(0.54,1.18)\end{array}$ & $\begin{array}{c}0.89 \\
(0.62,1.28)\end{array}$ & $\begin{array}{c}0.85 \\
(0.58,1.27)\end{array}$ & $\begin{array}{c}0.95 \\
(0.67,1.34)\end{array}$ & RT+XELIRI & & & & \\
\hline $\begin{array}{c}1.11 \\
(0.79,1.57)\end{array}$ & $\begin{array}{c}1.25 \\
(0.86,1.80)\end{array}$ & $\begin{array}{c}1.20 \\
(0.84,1.70)\end{array}$ & $\begin{array}{c}1.33 \\
(0.90,1.96)\end{array}$ & $\begin{array}{c}1.40 \\
(0.83,2.35)\end{array}$ & $\mathrm{RT}+\mathrm{IS}$ & & & \\
\hline $\begin{array}{c}1.06 \\
(0.82,1.36)\end{array}$ & $\begin{array}{c}1.18 \\
(0.89,1.57)\end{array}$ & $\begin{array}{c}1.14 \\
(0.88,1.47)\end{array}$ & $\begin{array}{c}1.26 \\
(0.93,1.72)\end{array}$ & $\begin{array}{c}1.33 \\
(0.84,2.11)\end{array}$ & $\begin{array}{c}0.95 \\
(0.62,1.46)\end{array}$ & RT+UFT & & \\
\hline $\begin{array}{c}0.63 \\
(0.32,1.24)\end{array}$ & $\begin{array}{c}0.70 \\
(0.36,1.37)\end{array}$ & $\begin{array}{c}0.67 \\
(0.34,1.33)\end{array}$ & $\begin{array}{c}0.75 \\
(0.39,1.44)\end{array}$ & $\begin{array}{c}0.79 \\
(0.45,1.37)\end{array}$ & $\begin{array}{c}0.56 \\
(0.26,1.21)\end{array}$ & $\begin{array}{c}0.59 \\
(0.29,1.22)\end{array}$ & RT+XELIRI+CET & \\
\hline $\begin{array}{c}1.06 \\
(1.00,1.12)\end{array}$ & $\begin{array}{c}1.18 \\
(1.03,1.36)\end{array}$ & $\begin{array}{c}1.14 \\
(1.04,1.25)\end{array}$ & $\begin{array}{c}1.27 \\
(1.05,1.52)\end{array}$ & $\begin{array}{c}1.34 \\
(0.90,1.97)\end{array}$ & $\begin{array}{c}0.95 \\
(0.67,1.35)\end{array}$ & $\begin{array}{c}1.00 \\
(0.78,1.29)\end{array}$ & $\begin{array}{c}1.70 \\
(0.86,3.35)\end{array}$ & RT \\
\hline
\end{tabular}

C

\begin{tabular}{|c|c|c|c|c|c|c|c|}
\hline $\mathrm{RT}+5-\mathrm{FU}$ & & & & & & & \\
\hline $\begin{array}{c}0.91 \\
(0.74,1.11)\end{array}$ & $\mathrm{RT}+\mathrm{CAPE}$ & & & & & & \\
\hline $\begin{array}{c}0.98 \\
(0.86,1.11)\end{array}$ & $\begin{array}{c}1.08 \\
(0.85,1.37)\end{array}$ & RT+FOLFOX & & & & & \\
\hline $\begin{array}{c}0.78 \\
(0.61,1.00)\end{array}$ & $\begin{array}{c}0.86 \\
(0.74,1.00)\end{array}$ & $\begin{array}{c}0.76 \\
(0.58,1.01)\end{array}$ & RT+XELOX & & & & \\
\hline $\begin{array}{c}0.72 \\
(0.36,1.43)\end{array}$ & $\begin{array}{c}0.79 \\
(0.41,1.54)\end{array}$ & $\begin{array}{c}0.71 \\
(0.35,1.42)\end{array}$ & $\begin{array}{c}0.92 \\
(0.49,1.75)\end{array}$ & RT+XELIRI & & & \\
\hline $\begin{array}{c}0.94 \\
(0.37,2.41)\end{array}$ & $\begin{array}{c}1.04 \\
(0.40,2.72)\end{array}$ & $\begin{array}{c}0.92 \\
(0.36,2.39)\end{array}$ & $\begin{array}{c}1.21 \\
(0.45,3.20)\end{array}$ & $\begin{array}{c}1.32 \\
(0.41,4.25)\end{array}$ & $R T+I S$ & & \\
\hline $\begin{array}{c}1.15 \\
(0.83,1.59)\end{array}$ & $\begin{array}{c}1.27 \\
(0.87,1.86)\end{array}$ & $\begin{array}{c}1.13 \\
(0.80,1.60)\end{array}$ & $\begin{array}{c}1.48 \\
(0.98,2.23)\end{array}$ & $\begin{array}{c}1.62 \\
(0.75,3.47)\end{array}$ & $\begin{array}{c}1.25 \\
(0.46,3.44)\end{array}$ & $\mathrm{RT}+\mathrm{UFT}$ & \\
\hline $\begin{array}{c}0.99 \\
(0.93,1.06)\end{array}$ & $\begin{array}{c}1.10 \\
(0.89,1.35)\end{array}$ & $\begin{array}{c}0.97 \\
(0.84,1.12)\end{array}$ & $\begin{array}{c}1.27 \\
(0.99,1.65)\end{array}$ & $\begin{array}{c}1.39 \\
(0.70,2.78)\end{array}$ & $\begin{array}{c}1.08 \\
(0.41,2.81)\end{array}$ & $\begin{array}{c}0.86 \\
(0.62,1.20)\end{array}$ & RT \\
\hline
\end{tabular}

Figure 2 ORs for pCR (A). HRs for DFS (B) and OS (C). Results with statistical significance are bold. pCR, pathological complete response rate; DFS, disease-free survival; OS, overall survival. 


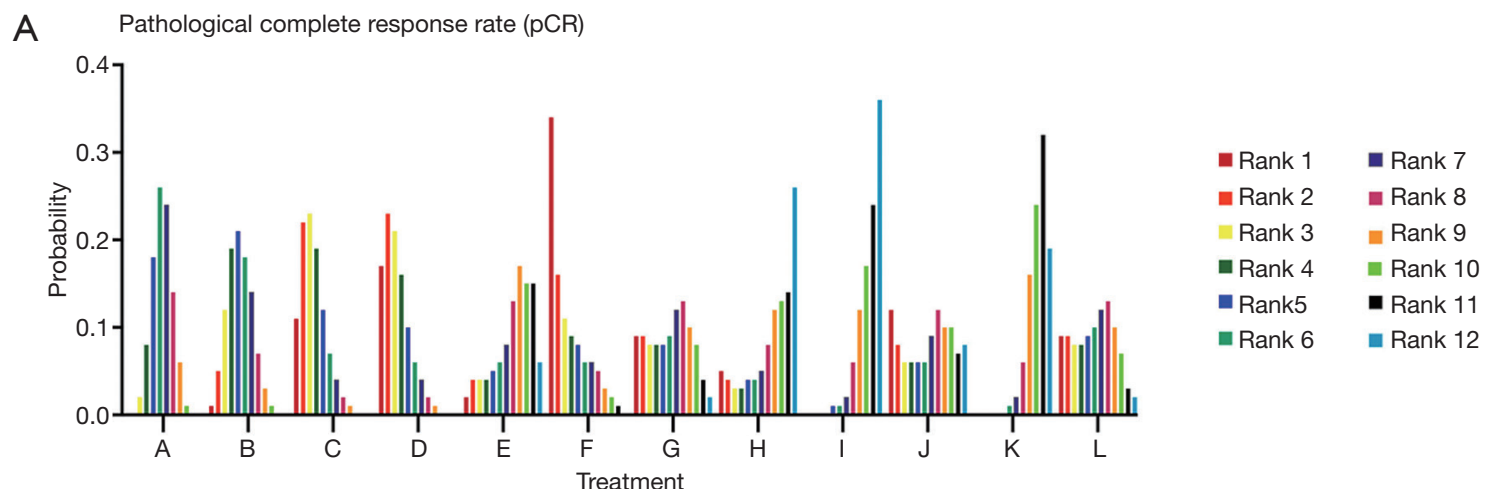

B Disease-free survival (DFS)

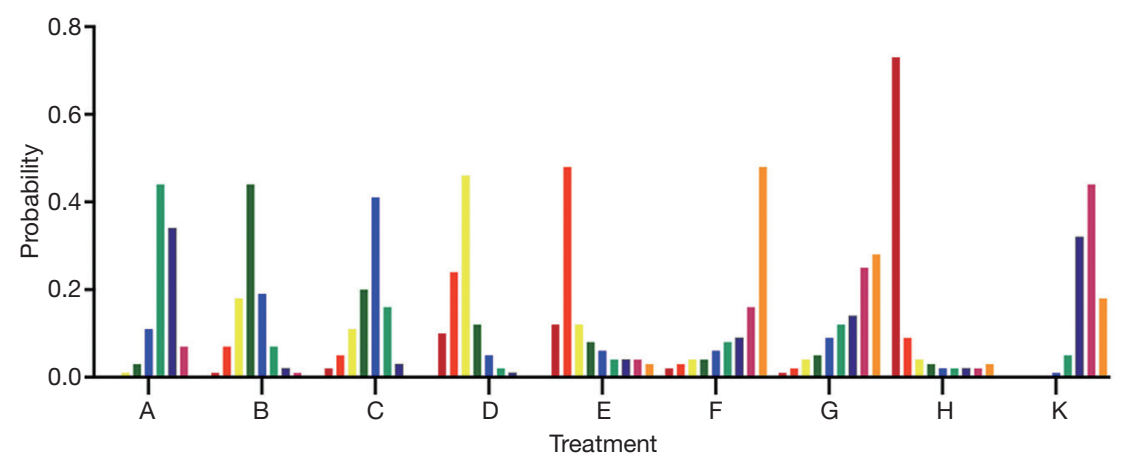

$$
\begin{aligned}
& \text { - Rank } 1 \text { Rank } 7 \\
& \text { - Rank } 2 \text { - Rank } 8 \\
& \text { Rank } 3 \text { Rank } 9
\end{aligned}
$$

C Overall survival (OS)

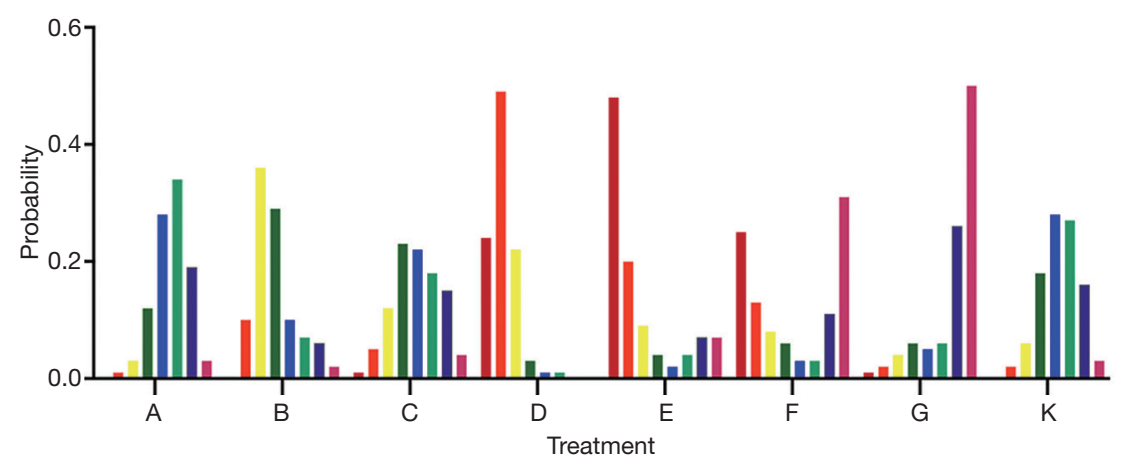

- Rank 1 Rank 7

- Rank 2 - Rank 8

$\square$ Rank 3

- Rank 4

- Rank5

nank 6

Figure 3 Rankograms for pCR (A), DFS (B) and OS (C). The figure shows the probability of each treatment from the best to the worst. The height of the columns for "rank 1, rank 2, etc." refers to the probability of each rank. If the height of the column belonging to intervention $\mathrm{X}$ painted in the color of "rank 1" was 0.4 , it means the probability of interval X to rank first was $40 \%$. Treatment legend: A: RT+5-Fu, B: RT+CAPE, C: RT+FOLFOX, D: RT+XELOX, E: RT+XELIRI, F: RT+IS, G: RT+UFT, H: RT+XELIRI+CET, I: FOLFOX, J: RT+CAPE+BEV, K: RT, L: RT+FOLFIRI. pCR, pathological complete response rate; DFS, disease-free survival; OS, overall survival.

results of ACCORD-12, STAR-01, NSABP R-04, CAO/ ARO/AIO-04, and PTEACC-6 trials, RT+XELOX did not show significantly better outcomes for except a slight benefit. For example, only the CAO/ARO/AIO-04 trial found that the addition of oxaliplatin had benefits in 3-year DFS (HR $=0.79,95 \%$ CI, 0.64-0.98, $\mathrm{P}=0.03$ ) (12). The
pCR of the addition of oxaliplatin was slightly higher in the ACCORD-12 trial (19\% vs. $14 \%, \mathrm{P}=0.09)$ and CAO/ ARO/AIO-04 trial $(17 \%$ vs. $13 \%, \mathrm{P}=0.04)(13,16)$. The advantages of RT+XELOX in our study may be due to the accumulation of sample size.

With the development of target therapy, attempts to 
A

\begin{tabular}{|c|c|c|c|c|c|c|c|c|c|c|c|c|}
\hline Intervention & $\begin{aligned} & R T \\
+ & 5-\mathrm{FU}\end{aligned}$ & $\begin{aligned} & \mathrm{RT} \\
+ & \mathrm{CAPE}\end{aligned}$ & $\begin{array}{c}\mathrm{RT} \\
+ \text { FOLFOX }\end{array}$ & $\begin{aligned} & \text { RT } \\
&+ \text { XELOX }\end{aligned}$ & $\begin{array}{c}\text { RT } \\
+ \text { +XELIRI }\end{array}$ & $\begin{array}{l}\text { RT } \\
+ \text { IS }\end{array}$ & $\begin{aligned} & R T \\
+ & \text { UFT }\end{aligned}$ & $\begin{array}{c}\text { RT }+ \text { XELIRI } \\
+ \text { +CET }\end{array}$ & FOLFOX & $\begin{array}{c}\mathrm{RT}+\mathrm{CAPE} \\
+\mathrm{BEV}\end{array}$ & RT & $\begin{array}{c}\mathrm{RT} \\
+ \text { FOLFIRI }\end{array}$ \\
\hline pCR & $50.91 \%$ & $62.27 \%$ & $77.91 \%$ & $79.18 \%$ & $35.36 \%$ & $78.27 \%$ & $54.27 \%$ & $30.09 \%$ & $12.82 \%$ & $49.27 \%$ & $15.27 \%$ & $55.18 \%$ \\
\hline os & $30.23 \%$ & $56.21 \%$ & $39.09 \%$ & $83.49 \%$ & $78.06 \%$ & $54.34 \%$ & $25.84 \%$ & / & / & / & $32.75 \%$ & / \\
\hline
\end{tabular}

B

$$
\text { Pathological complete response rate }(\mathrm{pCR})
$$

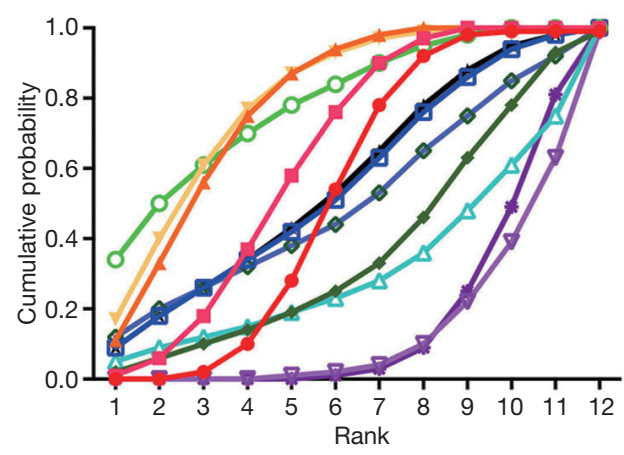

$$
\begin{aligned}
& \multimap \mathrm{RT}+5-\mathrm{FU} \\
& \rightarrow \text { RT+CAPE } \\
& - \text { RT+FOLFOX } \\
& \rightarrow \text { RT+XELOX } \\
& \leftarrow \text { RT+XELIRI } \\
& -O-R T+I S \\
& \text { RT+UFT } \\
& \triangle \mathrm{RT}+\mathrm{XELIRI+CET} \\
& \rightarrow \text { FOLFOX } \\
& \multimap \text { RT+CAPE+BEV } \\
& \because-\mathrm{RT} \\
& \text { - RT+FOLFIRI } \\
& \leadsto \mathrm{RT}+5-\mathrm{FU}
\end{aligned}
$$$$
\text { C }
$$$$
\text { Disease-free Survival (DFS) }
$$

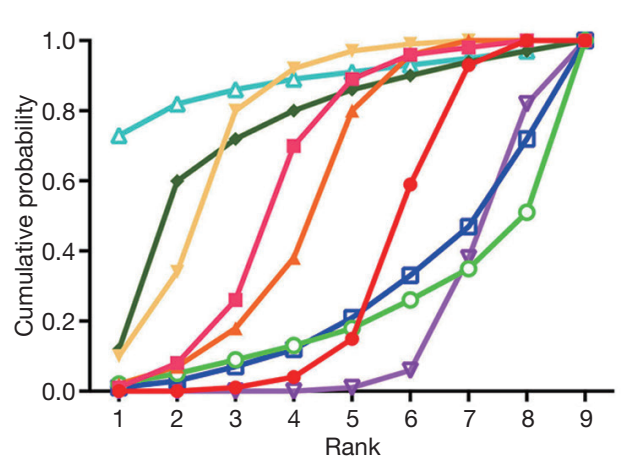

D
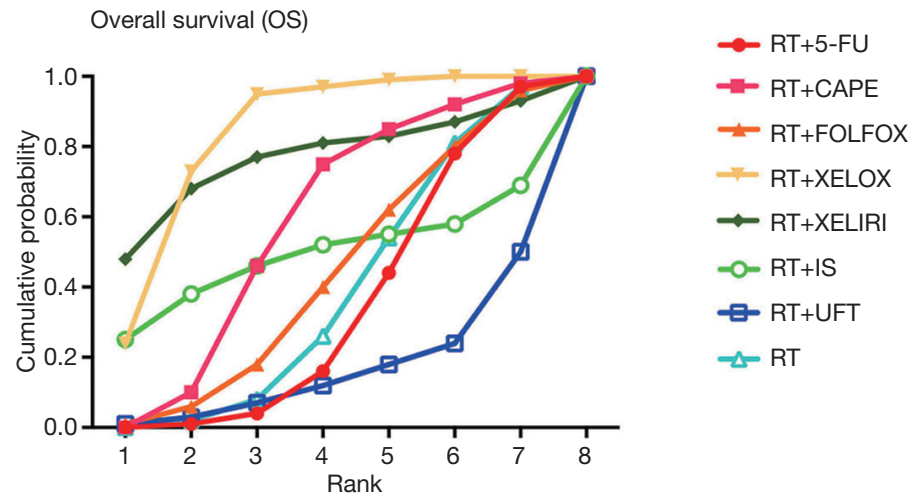

Figure 4 Value of SUCRA (A) and plots of SUCRA for pCR (B), DFS (C) and OS (D). SUCRA, surface under the cumulative ranking curve analysis; pCR, pathological complete response rate; DFS, disease-free survival; OS, overall survival. 

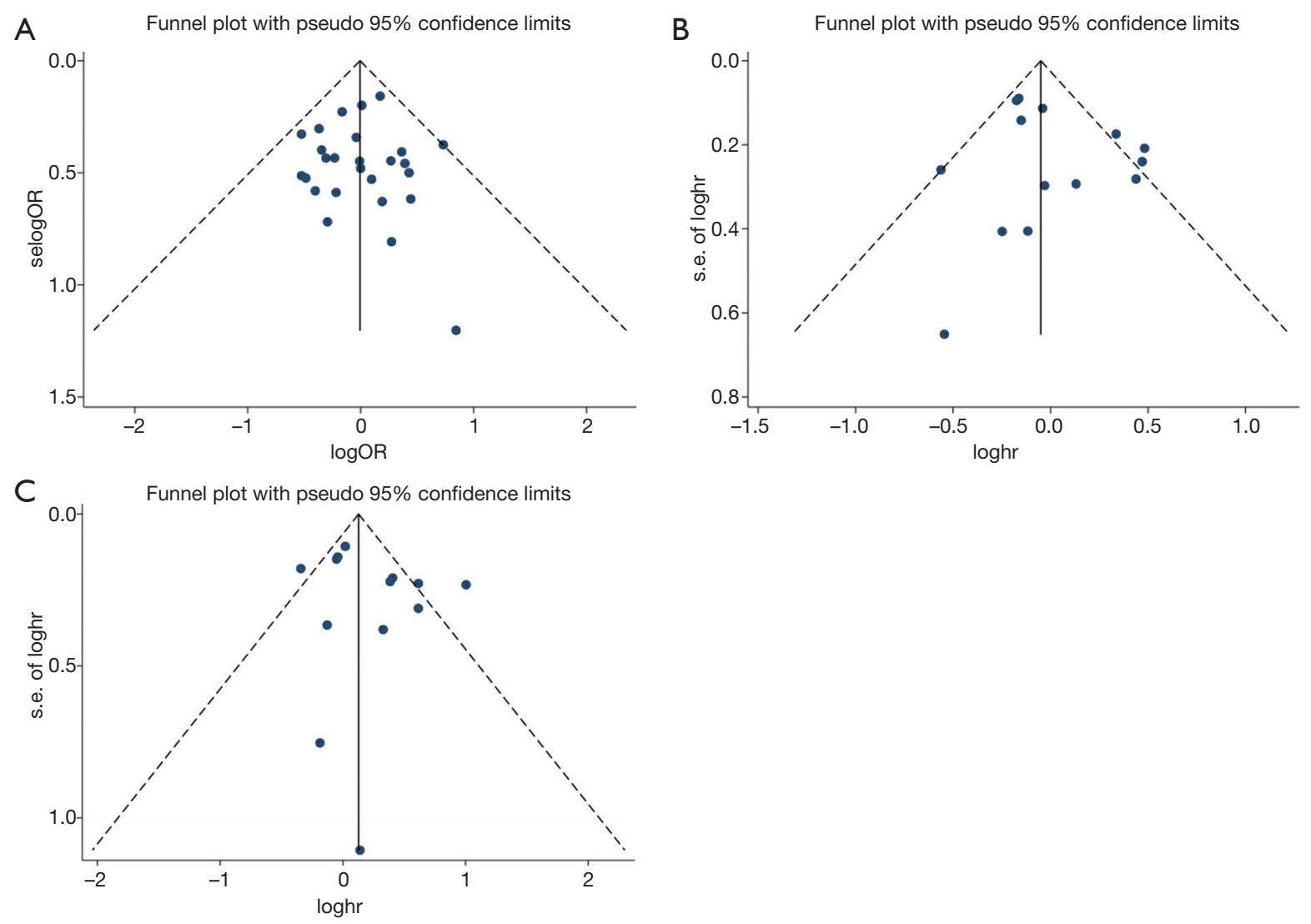

Figure 5 Funnel plots for pCR (A), DFS (B) and OS (C). pCR, pathological complete response rate; DFS, disease-free survival; OS, overall survival.

combine epidermal growth factor receptor (EGFR) and vascular endothelial growth factor (VEGF) monoclonal antibodies with chemoradiotherapy were made for better efficacy. Our study showed that RT+XELIRI+CET ranked first in DFS but inferior in pCR. In some practice, addition of anti-EGFR in chemoradiotherapy confers a pCR of $19-25 \%(41,42)$. In addition, bevacizumab-containing chemoradiotherapy had a pCR of $18.4-19.2 \%(43,44)$. Nonetheless, there was no powerful evidence about adding monoclonal antibodies to chemoradiotherapy in RCTs.

Owing to the side effects of radiotherapy, chemotherapy alone may be an option. In the FOWARC trial, clinicians made an attempt to get rid of radiotherapy (30). Besides, immunotherapy in deficient-mismatch repair (d-MMR) and microsatellite instability-high (MSI-H) patients, proved to have desirable outcomes, and potentially may be used to treat LARC patients. In the NICHE study, ipilimumab and nivolumab as neoadjuvant therapies for nonmetastatic colon cancer achieved a major response rate of $100 \%$ and pCR of $57 \%$ (4/7) in seven d-MMR patients, indicating that neoadjuvant immunotherapy may be tried in LARC patients with $\mathrm{d}-\mathrm{MMR}$ in the future (45).

There are several limitations of the network metaanalysis. Firstly, in the randomized, multicenter, noninferiority, phase 3 trial conducted by Hofheinz et al., HR of DFS was calculated by the data of both adjuvant and neoadjuvant cohorts and specific data about the neoadjuvant cohort were not mentioned in the article (20). It may lead to some bias in the final results. Secondly, the toxicity of these treatments was not analyzed on account of the insufficient reports. Thirdly, the use of the fixed-effects model ignored the heterogeneity between studies, which may lead to overestimated results. Some outcomes without full-text manuscripts were not included.

\section{Conclusions}

This network meta-analysis aimed to assess the regimens of preoperative chemoradiotherapy in LARC, and a combination of oxaliplatin, capecitabine together with 
radiotherapy $(\mathrm{RT}+\mathrm{XELOX})$ is likely to be the best treatment option with a comprehensive curative effect currently. Despite the regimen being not recommended, it may have a potential in the future based on more evidence.

\section{Acknowledgments}

Funding: This work was supported by the National Natural Science Foundation of China (grants 81572389, 81871944) and Jiangsu Province Key Medical Talents (grant ZDRCA2016026).

\section{Footnote}

Reporting Checklist: The authors have completed the PRISMA reporting checklist. Available at http://dx.doi. org/10.21037/tcr-20-683

Peer Review File: Available at http://dx.doi.org/10.21037/tcr20-683

Conflicts of Interest: All authors have completed the ICMJE uniform disclosure form (available at http://dx.doi. org/10.21037/tcr-20-683). The authors have no conflicts of interest to declare.

Ethical Statement: The authors are accountable for all aspects of the work in ensuring that questions related to the accuracy or integrity of any part of the work are appropriately investigated and resolved. This meta-analysis adheres to the requirements of the Preferred Reporting Items for Systematic Reviews and Meta-Analyses (PRISMA) statement. An IRB approval and informed consent of the patients were not required for this network meta-analysis.

Open Access Statement: This is an Open Access article distributed in accordance with the Creative Commons Attribution-NonCommercial-NoDerivs 4.0 International License (CC BY-NC-ND 4.0), which permits the noncommercial replication and distribution of the article with the strict proviso that no changes or edits are made and the original work is properly cited (including links to both the formal publication through the relevant DOI and the license). See: https://creativecommons.org/licenses/by-nc-nd/4.0/.

\section{References}

1. National Comprehensive Cancer Network Guidelines,
Rectal Cancer, Version 3.2018. Available online: http: // www.nccn.org. Accessed August 7, 2018.

2. Higgins JPT, Altman DG, Sterne JAC. Cochrane Handbook for Systematic Reviews of Interventions. In: Higgins J, Green S, editors. Chapter 8: Assessing risk of bias in included studies. Version 5.1.0. 2011. Available online: www.cochrane-handbook.org

3. Wells GA, Shea B, O'Connell D, et al. The NewcastleOttawa Scale (NOS) for assessing the quality of nonrandomised studies in meta-analyses. Available online: http://www.ohri.ca/programs/clinical_epidemiology/ oxford.asp

4. Bernardinelli L, Clayton D, Pascutto C, et al. Bayesian analysis of space-time variation in disease risk. Available online: https://onlinelibrary.wiley.com/doi/abs/10.1002/ sim. 4780142112

5. Carlin JB. Meta-analysis for $2 \times 2$ tables: a Bayesian approach. Stat Med 1992;11:141-58.

6. Dias S, Welton NJ, Caldwell DM, et al. Checking consistency in mixed treatment comparison meta-analysis. Stat Med 2010;29:932-44.

7. Brooks S, Gelman A. General Methods for Monitoring Convergence of Iterative Simulations. J Comput Graph Stat 1998;7:434-55.

8. van Valkenhoef G, Lu G, de Brock B, et al. Automating network meta-analysis. Res Synth Methods 2012;3:285-99.

9. Salanti G, Ades AE, Ioannidis JP. Graphical methods and numerical summaries for presenting results from multipletreatment meta-analysis: an overview and tutorial. J Clin Epidemiol 2011;64:163-71.

10. Duval S, Tweedie R. Trim and fill: A simple funnel-plotbased method of testing and adjusting for publication bias in meta-analysis. Biometrics 2000;56:455-63.

11. Fokas E, Ströbel P, Fietkau R, et al. Tumor Regression Grading After Preoperative Chemoradiotherapy as a Prognostic Factor and Individual-Level Surrogate for Disease-Free Survival in Rectal Cancer. J Natl Cancer Inst 2017;109:10.1093/jnci/djx095.

12. Rödel C, Graeven U, Fietkau R, et al. Oxaliplatin added to fluorouracil-based preoperative chemoradiotherapy and postoperative chemotherapy of locally advanced rectal cancer (the German CAO/ARO/AIO-04 study): final results of the multicentre, open-label, randomised, phase 3 trial. Lancet Oncol 2015;16:979-89.

13. Rödel C, Liersch T, Becker H, et al. Preoperative chemoradiotherapy and postoperative chemotherapy with fluorouracil and oxaliplatin versus fluorouracil alone in locally advanced rectal cancer: initial results of the German 
CAO/ARO/AIO-04 randomised phase 3 trial. Lancet Oncol 2012;13:679-87.

14. Azria D, Doyen J, Jarlier M, et al. Late toxicities and clinical outcome at 5 years of the ACCORD 12/0405-PRODIGE 02 trial comparing two neoadjuvant chemoradiotherapy regimens for intermediate-risk rectal cancer. Ann Oncol 2017;28:2436-42.

15. Gérard JP, Azria D, Gourgou-Bourgade S, et al. Clinical outcome of the ACCORD 12/0405 PRODIGE 2 randomized trial in rectal cancer. J Clin Oncol 2012;30:4558-65.

16. Gérard JP, Azria D, Gourgou-Bourgade S, et al. Comparison of two neoadjuvant chemoradiotherapy regimens for locally advanced rectal cancer: results of the phase III trial ACCORD 12/0405-Prodige 2. J Clin Oncol 2010;28:1638-44.

17. Jung M, Shin SJ, Koom WS, et al. A Randomized Phase 2 Study of Neoadjuvant Chemoradiaton Therapy with 5-Fluorouracil/Leucovorin or Irinotecan/S-1 in Patients With Locally Advanced Rectal Cancer. Int J Radiat Oncol Biol Phys 2015;93:1015-22.

18. Wong SJ, Moughan J, Meropol NJ, et al. Efficacy endpoints of radiation therapy group protocol 0247: a randomized, phase 2 study of neoadjuvant radiation therapy plus concurrent capecitabine and irinotecan or capecitabine and oxaliplatin for patients with locally advanced rectal cancer. Int J Radiat Oncol Biol Phys 2015;91:116-23.

19. Wong SJ, Winter K, Meropol NJ, et al. Radiation Therapy Oncology Group 0247: a randomized Phase II study of neoadjuvant capecitabine and irinotecan or capecitabine and oxaliplatin with concurrent radiotherapy for patients with locally advanced rectal cancer. Int J Radiat Oncol Biol Phys 2012;82:1367-75.

20. Hofheinz RD, Wenz F, Post S, et al. Chemoradiotherapy with capecitabine versus fluorouracil for locally advanced rectal cancer: a randomised, multicentre, non-inferiority, phase 3 trial. Lancet Oncol 2012;13:579-88.

21. Martijnse IS, Dudink RL, Kusters M, et al. T3+ and T4 rectal cancer patients seem to benefit from the addition of oxaliplatin to the neoadjuvant chemoradiation regimen. Ann Surg Oncol 2012;19:392-401.

22. Braendengen M, Tveit KM, Berglund A, et al. Randomized phase III study comparing preoperative radiotherapy with chemoradiotherapy in nonresectable rectal cancer. J Clin Oncol 2008;26:3687-94.

23. de la Torre A, García-Berrocal MI, Arias F, et al. Preoperative chemoradiotherapy for rectal cancer: randomized trial comparing oral uracil and tegafur and oral leucovorin vs. intravenous 5-fluorouracil and leucovorin. Int J Radiat Oncol Biol Phys 2008;70:102-10.

24. Gérard JP, Conroy T, Bonnetain F, et al. Preoperative radiotherapy with or without concurrent fluorouracil and leucovorin in T3-4 rectal cancers: results of FFCD 9203. J Clin Oncol 2006;24:4620-5.

25. Boulis-Wassif S, Gerard A, Loygue J, et al. Final results of a randomized trial on the treatment of rectal cancer with preoperative radiotherapy alone or in combination with 5-fluorouracil, followed by radical surgery. Trial of the European Organization on Research and Treatment of Cancer Gastrointestinal Tract Cancer Cooperative Group. Cancer 1984;53:1811-8.

26. Bosset JF, Collette L, Calais G, et al. Chemotherapy with preoperative radiotherapy in rectal cancer. $\mathrm{N}$ Engl J Med 2006;355:1114-23.

27. Kim SY, Shim EK, Yeo HY, et al. KRAS mutation status and clinical outcome of preoperative chemoradiation with cetuximab in locally advanced rectal cancer: a pooled analysis of 2 phase II trials. Int J Radiat Oncol Biol Phys 2013;85:201-7.

28. Singh K, Gupta MK, Seam RK, et al. A prospective randomized trial comparing capecitabine-based chemoradiotherapy with 5-FU-based chemoradiotherapy in neoadjuvant setting in locally advanced carcinoma rectum. Indian J Cancer 2017;54:347-51.

29. Wiśniowska K, Nasierowska-Guttmejer A, Polkowski $W$, et al. Does the addition of oxaliplatin to preoperative chemoradiation benefit c'T4 or fixed c'T3 rectal cancer treatment? A subgroup analysis from a prospective study. Eur J Surg Oncol 2016;42:1859-65.

30. Deng Y, Lan P, Wang L, et al. Modified FOLFOX6 With or Without Radiation Versus Fluorouracil and Leucovorin With Radiation in Neoadjuvant Treatment of Locally Advanced Rectal Cancer: Initial Results of the Chinese FOWARC Multicenter, Open-Label, Randomized ThreeArm Phase III Trial. J Clin Oncol 2016;34:3300-7.

31. Salazar R, Capdevila J, LaquenteB, et al. A randomized phase II study of capecitabine-based chemoradiation with or without bevacizumab in resectable locally advanced rectal cancer: clinical and biological features. BMC Cancer 2015;15:60.

32. Saha A, Ghosh SK, Roy C, et al. A randomized controlled pilot study to compare capecitabine-oxaliplatin with 5-FUleucovorin as neoadjuvant concurrent chemoradiation in locally advanced adenocarcinoma of rectum. J Cancer Res Ther 2015;11:88-93.

33. Aschele C, Cionini L, Lonardi S, et al. Primary tumor 
response to preoperative chemoradiation with or without oxaliplatin in locally advanced rectal cancer: pathologic results of the STAR-01 randomized phase III trial. J Clin Oncol 2011;29:2773-80.

34. Kim JS, Kim JS, Cho MJ, et al. Comparison of the efficacy of oral capecitabine versus bolus 5 -FU in preoperative radiotherapy of locally advanced rectal cancer. J Korean Med Sci 2006;21:52-7.

35. Mohiuddin M, Winter K, Mitchell E, et al. Randomized phase II study of neoadjuvant combined-modality chemoradiation for distal rectal cancer: Radiation Therapy Oncology Group Trial 0012. J Clin Oncol 2006;24:650-5.

36. McCarthy K, Pearson K, Fulton R, et al. Pre-operative chemoradiation for non-metastatic locally advanced rectal cancer. Cochrane Database Syst Rev 2012;12:CD008368.

37. Zhao N, Lin CJ, Wang Fei, et al. Short-course or longcourse radiation therapy as a part of a neoadjuvant regimen for stage II \& III rectal adenocarcinoma? Chin J Cancer Res 2019;31:849-52.

38. Petrelli F, Sgroi G, Sarti E, et al. Increasing the Interval Between Neoadjuvant Chemoradiotherapy and Surgery in Rectal Cancer: A Meta-analysis of Published Studies. Ann Surg 2016;263:458-64.

39. O'Connell MJ, Colangelo LH, Beart RW, et al. Capecitabine and oxaliplatin in the preoperative multimodality treatment of rectal cancer: surgical end points from National Surgical Adjuvant Breast and Bowel
Project trial R-04. J Clin Oncol 2014;32:1927-34.

40. Allegra CJ, Yothers G, O'Connell MJ, et al. Neoadjuvant 5-FU or Capecitabine Plus Radiation With or Without Oxaliplatin in Rectal Cancer Patients: A Phase III Randomized Clinical Trial. J Natl Cancer Inst 2015;107:djv248.

41. Jin T, Zhu Y, Luo JL, et al. Prospective phase II trial of nimotuzumab in combination with radiotherapy and concurrent capecitabine in locally advanced rectal cancer. Int J Colorectal Dis 2015;30:337-45.

42. Pinto C, Di Fabio F, Maiello E, et al. Phase II study of panitumumab, oxaliplatin, 5-fluorouracil, and concurrent radiotherapy as preoperative treatment in high-risk locally advanced rectal cancer patients (StarPan/STAR-02 Study). Ann Oncol 2011;22:2424-30.

43. Sadahiro S, Suzuki T, Tanaka A, et al. Phase II study of preoperative concurrentchemoradiotherapy with S-1 plus bevacizumab for locally advanced resectable rectal adenocarcinoma. Oncology 2015;88:49-56.

44. Kennecke H, Berry S, Wong R, et al. Pre-operative bevacizumab, capecitabine, oxaliplatin and radiation among patients with locally advanced or low rectal cancer: a phase II trial. Eur J Cancer 2012;48:37-45.

45. Chalabi M, Fanchi LF, Van den Berg JG, et al. Neoadjuvant ipilimumab plus nivolumab in early stage colon cancer. Ann Oncol 2018. doi: 10.1093/annonc/ mdy424.047
Cite this article as: Yu Z, Wang J, Xu L, Liu J, Chen X, Gu Y. Comparison of different chemoradiotherapy regimens for the preoperative treatment in patients with locally advanced rectal cancer: a network meta-analysis. Transl Cancer Res 2020;9(8):48574869. doi: 10.21037/tcr-20-683 